\title{
A study on local therapy technique that can be used in treatment of AIDS and some other diseases
}

\author{
Sameh Monir Abdou Desouki Salem \\ Gizan General Hospital, KSA, Saudi Arabia
}

\begin{abstract}
Introduction: Local therapy is treatment that is directed to a specific organ or limited area of the body. It can shorten the length of infection, reduce complications, and reduce the spread to other tissues or organs. In some cases, it may be more effective, more specific and better than systematic therapy, with fewer side effects. In this research, I tried to evaluate and develop the use and implementation of some of these local therapy medications in treatment of some diseases including acquired immunodeficiency syndrome (AIDS).

Material and methods: I used in this research direct face-to-face questionnaires to get my answers and data from adult people who experienced these diseases and therapies before. They provided me with all required information without identification data and without the need of any consent or legal permissions. Also I collected retrospectively registered data as nominal data to be easier in calculation and evaluation with a probability value $p<0.05$.

Results: I got some data and results with a significant difference, e.g. 103 (103 from 129 total sample $=79.84 \%$ ) patients with minor superficial wounds, who have an experience with these diseases and both of local and systematic therapies before, said that they had a faster recovery (by 2-3 days) if using a combination of a systematic antibiotic with a local antibiotic cream than using the same systematic antibiotic alone. Also, 4 patients (from a total sample of $6=66.66 \%$ ) with AIDS got a faster and better outcome in decreasing viral load if using a combination of systematic oral antiretroviral treatment with injectable intramuscular injection of antiretroviral treatment (oral zidovudine $300 \mathrm{mg}$ + lamivudine $150 \mathrm{mg}$ USP, one tablet once daily for 24 weeks and with injection (intramuscular dosing) antiretroviral therapy, as injectable cabotegravir $400 \mathrm{mg}$ + rilpivirine $600 \mathrm{mg}$ one vial dose every 4 weeks for 24 weeks).

Conclusions: The study revealed that we can use and develop some medications as an effective therapy in treatment of some diseases, including human immunodeficiency virus. I have suggested in this research a new additional way for treatment of AIDS, taking into account the possible side effects.
\end{abstract}

HIV AIDS Rev 2019; 18, 3: 193-198 DOI: https://doi.org/10.5114/hivar.2019.88196

Key words: local therapy, AIDS, HIV, CD4, bone marrow.

\section{Address for correspondence: Sameh Monir Abdou Desouki, Gizan General Hospital, KSA, Saudi Arabia,}

phone: 00966548331799, e-mail: sammon2002@yahoo.com
Article history:

Received: 08.03.2019

Received in revised form: 06.05.2019

Accepted: 07.05.2019

Available online: 17.06 .2019
International Journal of HIV-Related Problem

HIV \& AIDS

R e v i e w 


\section{Introduction}

Local therapy is a treatment that is directed to a specific organ or limited area of the body [1], and it is an important technique in therapy planning that could be used alone or with other types of therapy techniques, e.g. it can be used with systematic therapy, in treatments of some diseases or conditions. Also, it can shorten the length of infection, reduce complications, and reduce the spread to other tissues or organs. In other words, in many conditions, local therapy, as a route of drug or therapy administration, may be more effective and better than systematic therapy with fewer side effects.

Local therapy can be more specific and localized than systematic therapy, as it can reach specific tissues that systematic therapy medication cannot reach sufficiently, e.g. in treatment of some of minor superficial wounds, trauma, infectious skin diseases, cancer and acquired immunodeficiency syndrome (AIDS) (AIDS stands for acquired immune deficiency syndrome; it is also called advanced human immunodeficiency virus [HIV] infection or late-stage HIV), and also in cancer treatment such as surgery, radiation therapy, cryotherapy, laser therapy, and topical therapy [1] (as a lotion or cream that is applied locally to the skin).

The life cycle of HIV can be only about 1.5 days from viral entry into a cell, but the virus can be replicated, assembled, and released to infect other cells [2]. Although there are some antiretroviral regimens that in some cases can decrease the viral load to an undetectable amount in the blood or even may eradicate the whole virus in the blood for some time, there is no regimen that can eradicate all the virus from the entire body permanently $[3,4]$. This effect can be due to some reasons, as the regimen drug treatment could decrease the viral load to an undetectable amount in blood $(<50$ copies per $\mathrm{ml}$ ) [5] or even may eradicate the whole virus in specific tissue in which the regimen can reach sufficiently. But the regimen cannot work effectively in all tissues as it cannot reach them in a sufficient dose amount and for a sufficient time of treatment.

So, the antiretroviral therapy (ART) drugs cannot eradicate all the virus from all the other tissues and fluids where the virus exists in which it cannot reach in a sufficient drug concentration. HIV is not found only in blood, but also can be in all body fluids: plasma, tears, semen, vaginal secretion, rectal fluid, breast milk, cerebrospinal fluid [6] and bone marrow [7] In other words, about $10 \%$ of patients taking antiretroviral therapy who have an undetectable viral load in their blood have detectable levels of HIV in their cerebrospinal fluids $[8,9]$ and bone marrow can harbor HIV-infected cells [7], too.

I tried in this research to make a study of some of these medications that may be used locally in treatments of some diseases including AIDS. In this research, I focused on AIDS treatment in the cases that I have faced or have known during my work in private and in general hospital pharmacies. I found some results that may reveal that using some medications locally with or without the other medication techniques may cause better positive treatment outcomes and rapid recovery, with regard to some side effects, including AIDS.

\section{Material and methods}

During my work at hospital pharmacies (during November 2014 to June 2018) I noticed and collected some data (by direct face-to-face interview and computerized questionnaires [10]) from adult patients or people (aged about 21-65 years old) - those who had experienced these diseases and both local and systematic therapies before - and they provided some information about these issues according to the information that they obtained from their physicians and data recorded in their medical profile before. They informed me all required information without any identification data (such as names and address) which do not need any consent or legal permissions $[11,12]$. I collected those data as retrospectively registered data [13] and as nominal data [14] to be easier in calculation and evaluation (with a probability value $p<0.05$ ) $[15,16]$. I used some information from the internet too [17].

\section{Some observations in local therapy medications with examples}

Local therapy is a treatment that is directed to a specific organ or limited area of the body. The local therapy medications may produce very effective action alone or combined with other therapy techniques as with systematic therapy. Also, local therapy may shorten the length of infection, reduce complications, and reduce the spread to other tissues or organs. In other words, in many conditions, local therapy may be more effective and better with fewer side effects than systematic therapy, and it can reach into specific tissues that systematic therapy medication cannot reach sufficiently.

During my work in hospital pharmacies, I noticed and collected some information from adult people by directly asking them, and I obtained those retrospectively registered data and I put them in the form of nominal data so as to be easily calculated. The people were adult (21-65 years old), and they gave me all required information selectively with no confidential identification data, those not needing any consent or legal permissions $[11,12]$. They provided information about these issues according to the information that they have obtained from their physicians or recorded in their medical profile before, such as in the following examples.

\section{Use of plasma injections in harmed knee joint in osteoarthritis patients}

It was observed that injection of plasma, extracted from the patient himself, into the knee joint lumen causes an improvement and more rapid recovery than using systematic cortisone medications alone or any other medication alone.

In other words, 9 (from a total sample of $11=81.81 \%$ ) patients, with chronic osteoarthritis, have tried using all available medications in treatments of osteoarthritis including cortisone medications and analgesics as diclofenate sodium $50 \mathrm{mg}$ tablets twice daily or intramuscular injections but with no detectable or satisfactory response. They informed me that they detected a better improvement and faster recov- 
ery with injection of plasma with analgesics (mainly paracetamol $500 \mathrm{mg}$ tablets as needed), extracted from the patient himself, into the knee joint lumen, than using cortisone (5-20 mg prednisolone USP tablets 2-3 times daily for 2-4 weeks) medications alone or with any other medication, and with no unbearable side effects, after 3 months of injection of a single dose of $30 \mathrm{ml}$ of plasma in the knee joint. The rest of the patients ( 2 patients) said that there was no difference between the two techniques in positive outcomes.

\section{Use of medications in patients with minor superficial wounds}

One hundred and three (from a total sample of $129=$ $79.84 \%$ ) patients with minor superficial wounds, who have experience with these diseases and both local and systematic therapies before, said that they had a faster recovery (by 2-3 days) if using a combination of systematic antibiotic with local antibiotic cream than using the same systematic antibiotic alone (oral amoxicillin trihydrate $500 \mathrm{mg}$ tablets, USP, 3 time daily for 5-7 days and local gentamicin sulfate ointment USP, $0.1 \%$ twice daily for 5-10 daily), with no unbearable side effects, while the rest of the patients (26 patients) said that there was no difference between the two techniques in positive outcomes.

\section{Use of medications in patients with infectious skin diseases}

Patients (41 from a total sample of $63=65.07 \%$ ) with infectious skin diseases - mainly cellulitis - who have experience with these diseases and both local and systematic therapies before, had faster and better outcomes (faster by 2-3 days) if using a combination of a systematic antibiotic with a local antibiotic cream than using the same systematic antibiotic alone (oral amoxicillin trihydrate USP, $500 \mathrm{mg}$ tablets for 5-10 days and local gentamicin sulfate ointment USP, $0.1 \%$ twice daily for 5-10 days), with no unbearable side effects, while the rest of the patients (22 patients) said that there were no better positive outcomes between the techniques.

\section{Use of medications in patients with bacterial eye infection}

One hundred and four patients (from a total sample of $189=55.02 \%$ ) with bacterial eye infection diseases mainly acute bacterial conjunctivitis - who have experience with these diseases and both local and systematic therapies before, had faster (by 1-2 days) and better outcomes if using a combination of a systematic antibiotic with a local antibiotic cream than using the same local antibiotic alone (oral azithromycin monohydrate 500 gm USP, once daily for 3 days and local eye ointment $0.3 \%$ gentamycin USP, twice daily for 7-10 days). But they suffered from some extra mild side effects especially in the GIT (gastrointestinal tract), such as nausea, vomiting and GIT disturbances. The rest of the patients ( 85 patients) said that there was no difference between the techniques in positive outcomes.

\section{Use of medications in patients with viral eye infection}

Fifteen patients (from a total sample of $108=13.88 \%$ ) with viral eye infection diseases - mainly acute viral conjunctivitis - who have experience with these diseases and both local and systematic therapies before, had a faster (by 1-2 days) and better outcome if using a combination of a systematic antibiotic with a local antibiotic cream than using the same local antibiotic alone (oral corticosteroids $5 \mathrm{mg}$ prednisolone USP, once daily for 1 week and local eye drop $1 \%$ prednisolone USP, 4 times daily for 2 weeks). But they suffered from some extra mild to moderate side effects especially in the GIT such as nausea and vomiting, while the rest of the patients ( 93 patients) said that there was no difference between the techniques in positive outcomes.

\section{Use of medications in cancer treatment}

There were some patients ( 8 patients, 1 patient with leukemia, and 7 patients with prostatic tumor - but I could not detect all regimen details accurately) with local radiation therapy and chemical drug therapy who had a better response than radiation therapy alone or with antineoplastic drugs alone, and less cancer relapse or return if following these regimens for 3-6 months after complete recovery. But they suffered from some extra moderate to severe side effects especially in the GIT such as nausea and vomiting, and also anemia, neutropenia and leukopenia, after a period of treatment of about 2-12 weeks.

\section{Use of medications in AIDS treatments}

Four patients (from a total sample of $6=66.66 \%$; the patients had a viral load of 50-500 copies per ml) with AIDS had a faster and better outcome in decreasing viral load and increasing CD4 number (cluster of differentiation 4) if using a combination of systematic oral antiretroviral treatment with injectable intramuscular injection of antiretroviral treatment (oral zidovudine $300 \mathrm{mg}+$ lamivudine $150 \mathrm{mg}$ USP, one tablet once daily for 24 weeks and with injection antiretroviral therapy, as long acting injectable [18] (intramuscular dosing) cabotegravir $400 \mathrm{mg}+$ rilpivirine $600 \mathrm{mg}$ one vial dose every 4 weeks for 24 weeks). The rest of the patients ( 2 patients) said that there was no difference between the techniques in positive outcomes.

However, they suffered from some extra moderate to severe side effects such as nausea, severe blood problems including a decrease in red blood cells (anemia) and white blood cells, with a total period of treatment of 24 weeks. They told me that the virus returned about 8 weeks after stopping the high-dose regimen. One of them told me that although the virus disappeared to an undetectable number in the blood it was still found in higher numbers in other fluids such as cerebrospinal fluid. 


\section{AIDS drugs and treatment regimens}

There are many different types of antiretroviral drugs (ART) and they work in different ways. In most antiretroviral regimens there is usually more than one drug from different group types $[4,19]$, to prevent resistance and to give better results. However, usually we start HIV treatment by combination such as TDF (tenofovir), either 3TC (lamivudine) or FTC (emtricitabine), and EFV (efavirenz) [4, 19].

There are a lot of antiretroviral drugs, and they can be combined in different ways. The World Health Organization (WHO) has recommended a combination of antiretroviral drugs for people starting HIV treatment, such as the following regimens:

- tenofovir/emtricitabine and raltegravir,

- tenofovir/emtricitabine and dolutegravir,

- tenofovir disoproxil fumarate (300 mg) + efavirenz (600 mg) + emtricitabine (200 mg),

- abacavir/lamivudine (two NRTIs) [20],

- cobicistat, darunavir ethanolate,

- cabotegravir $30 \mathrm{mg}$, abacavir $600 \mathrm{mg}$, 3TC $300 \mathrm{mg}$ [21]. The regimen and treatment plan can be determining according to many factors such as patient conditions (pregnant, adult, child), virus load number, disease stage, disease severity, virus and medications resistance $[22,23]$.

And there are many drugs for AIDS treatment $[3,24,25]$ such as:

1. Nucleoside/nucleotide reverse transcriptase inhibitors (NRTIs): force the HIV virus to use faulty versions of building blocks so infected cells cannot make more HIV (e.g. abacavir, or ABC [Ziagen], tenofovir disoproxil fumarate, or TDF [Viread], zidovudine or ZDV [Retrovir], emtricitabine [FTC], lamivudine [3TC]).

2. Non-nucleoside reverse transcriptase inhibitors (NNRTIs): bind to a specific protein so the HIV virus cannot make copies of itself, similar to jamming a zipper (e.g. delavirdine or DLV [Rescripor], nevirapine or NVP [Viramune], rilpivirine or RPV [Edurant], doravirine [DOR], efavirenz [EFV], etravirine [ETR]).

3. Protease inhibitors (PIs): these drugs block a protein that infected cells need to put together new HIV virus particles (e.g. atazanavir or ATV [Reyataz], tipranavir or TPV [Aptivus], darunavir [darunavir ethanolate, DRV], fosamprenavir [fosamprenavir calcium, FOS-APV, FPV], ritonavir [RTV]).

4. Fusion inhibitors: unlike NRTIs, NNRTIs, and PIs which work on infected cells - these drugs help block HIV from getting inside healthy cells in the first place (e.g. enfuvirtide, or ENF or T-20 [Fuzeon]).

5. CCR5 antagonist: it stops HIV before it gets inside a healthy cell, but in a different way than fusion inhibitors. It blocks a specific kind of "hook" on the outside of certain cells so the virus cannot plug in. (e.g. maraviroc, or MVC [Selzentry]).

6. Integrase inhibitors: these stop HIV from making copies of itself by blocking a key protein that allows the virus to put its DNA into the healthy cell's DNA. They are also called integrase strand transfer inhibitors (INSTIs) (e.g. bictegravir or BIC [combined with other drugs as Biktarvy], dolutegravir or DTG [Tivicay], elvitegravir or EVG [Vitekta], raltegravir or RAL [Isentress]).

7. Post-attachment inhibitors: block CD4 receptors on the surface of certain immune cells that HIV needs to enter the cells. e.g. ibalizumab-uiyk (Hu5A8, IBA, Ibalizumab, TMB-355, TNX-355).

8. Pharmacokinetic enhancers: increase the effectiveness of an HIV medicine included in an HIV regimen, e.g. cobicistat (COBI).

\section{Results}

In using plasma injections in the harmed knee joint in osteoarthritis patients, use of antibiotics locally besides systematic drugs in patients with superficial wounds, using medications in viral and bacterial eye infection, using medications and radiation in cancer treatments, I found that different percentages of patients with these conditions had faster and better outcomes if using a combination of a systematic antibiotic with a local therapy regimen than when using the same local therapy alone. But there are some extra side effects with various degrees of severity.

In AIDS treatment, using a high dose of antiretroviral drugs, or using injection antiretroviral therapy with ordinal oral drugs doses, could cause some improvements and a decrease in viral load, after 12-24 weeks of treatments with normal recommended doses, but they could cause additional and worse side effects (Table 1).

\section{Discussion}

Local therapy is a treatment that is directed to a specific organ or limited area of the body. Local therapy medications may produce very effective action alone or combined with other therapy techniques such as with systematic therapy. Also, local therapy may shorten the length of infection, reduce complications, reduce the spread to other tissues or organs, and in some cases (including some viral infectious diseases) may be more effective, with fewer side effects and better than systematic therapy, and it can reach into specific tissues that systematic therapy medication cannot reach sufficiently [1].

In using medications in AIDS treatments, HIV is found not only in blood, but also can be in all body fluids, such as plasma, tears, semen, vaginal secretion, rectal fluid, breast milk, cerebrospinal fluid [6], and bone marrow [7]. So, we need to make these antiretroviral regimen drugs reach all these tissues and all fluids where the virus exists in a sufficient dose at the same time for a specific period of time of treatment, to prevent the virus recovering from any cell to any other cell or from tissue to tissue.

We can achieve these goals if we create new antiretroviral regimen of drugs that have these desirable properties, like if it can reach all needed tissues and fluids where 
Table 1. Summary of results

\begin{tabular}{|c|c|c|c|}
\hline $\begin{array}{l}\text { Patient said about } \\
\text { the regimen/condition }\end{array}$ & $\begin{array}{l}\text { Combination of systematic with local } \\
\text { therapy regimen is better }\end{array}$ & $\begin{array}{c}\text { Combination regimen } \\
\text { is not better than the other } \\
\text { monotherapy regimen technique }\end{array}$ & $\begin{array}{l}\text { Side effects } \\
\text { degree }\end{array}$ \\
\hline $\begin{array}{l}\text { Using plasma injections in knee } \\
\text { joint in osteoarthritis patients }\end{array}$ & $\begin{array}{l}9 \\
\text { (9 from } 11 \text { total sample }=81.81 \%) \\
\text { Plasma with analgesics (mainly } \\
\text { paracetamol tablets as needed) }\end{array}$ & $\begin{array}{l}2 \\
\text { Like cortisone and diclofenate } \\
\text { sodium oral and injection }\end{array}$ & None \\
\hline $\begin{array}{l}\text { Using medications in patients } \\
\text { with minor superficial wounds }\end{array}$ & $\begin{array}{c}103 \\
\text { (103 from } 129 \text { total sample }=79.84 \%)\end{array}$ & 26 & None \\
\hline $\begin{array}{l}\text { Using medications in patients } \\
\text { with infectious skin diseases }\end{array}$ & $\begin{array}{c}41 \\
(41 \text { from } 63 \text { total sample }=65.07)\end{array}$ & 22 & Mild \\
\hline $\begin{array}{l}\text { Using medications in bacterial } \\
\text { eye infection }\end{array}$ & $\begin{array}{c}104 \\
(104 \text { from } 189 \text { total sample }=55.02 \%)\end{array}$ & 85 & Mild \\
\hline $\begin{array}{l}\text { Using medications in viral eye } \\
\text { infection }\end{array}$ & $\begin{array}{l}15 \\
(15 \text { from } 108 \text { total sample }=13.88 \%)\end{array}$ & 93 & Moderate \\
\hline $\begin{array}{l}\text { Using medications in AIDS } \\
\text { treatments }\end{array}$ & $\begin{array}{c}4 \\
\text { (4 from } 6 \text { total sample }=66.66 \%) \\
\text { They used an extra intramuscular drug } \\
\text { or used excess dose }\end{array}$ & 2 & $\begin{array}{l}\text { Moderate - } \\
\text { severe }\end{array}$ \\
\hline
\end{tabular}

the virus exists, at the same time for a sufficient dose and for a sufficient period of treatment., or by other ways, such as by direct injection of the desired antiretroviral regimen drugs to all of the desirable tissue and fluids where the virus exists, at the same time for a sufficient dose and for a sufficient period of treatment. And we need to perform suitable clinical trials to achieve these aims (such as cohort studies) [26, 27], if these antiretroviral regimen drugs can decrease or even eliminate the virus from one tissue or fluid such as blood or vaginal secretions, so it is expected to eliminate the whole virus (in a shorter time and with better positive outcomes) from all the other fluids if given in all the fluids where the virus exist, at the same time, for a sufficient dose and for a sufficient period of treatment, with regard to the side effects, pharmacotherapeutic issues, and after taking into consideration drug activity, virus and the patient condition in both cerebral fluid and bone marrow and all body fluids in which the virus could exist, and also analysis and evaluation of all needed information, such as the concentration of the antiretroviral regimen drugs in both cerebrospinal fluid and bone marrow, and all body fluids and tissues, monitoring of drug concentration in both blood and all other fluids, drug toxicity, and dose monitoring during all the treatment course.

There are many studies that discuss these issues, such as those I have mentioned in this research with more details, but I failed to find a study that has dealt with these issues about AIDS treatments as I did in this research.

\section{Conclusions}

The study revealed that the use of local therapy techniques could be useful and give a better outcome in a lot of therapy regimens, and it can shorten the length of infection, reduce complications, reduce the spread to other tissues, decrease disease or condition severity, decrease microorganism numbers rapidly and effectively in some diseases including AIDS, that may be treated with this technique better with the desired positive outcomes including viral load and time of response, with regard to the possible side effects and all pharmacotherapeutic issues related to the drugs, virus and the patient condition.

\section{Acknowledgement}

The author expresses a deep sense of gratitude and acknowledges everybody from different nations and cultures around the world for helping him in his research.

\section{Availability of data and material}

All data are recorded in patients' medical profile before. Patients provided all required information selectively without any identification data (such as names and address) which do not need any consent or legal permissions.

\section{Conflict of interest}

The author declares no potential conflicts of interest with respect to the research, authorship, and/or publication of this article.

\section{References}

1. National Cancer Institute. Available at: https://www.cancer.gov/ publications/dictionaries/cancer-terms/def/local-therapy (Accessed: 18.01.2019). 
2. Perelson AS, Neumann AU, Markowitz M, et al. HIV-1 dynamics in vivo: virion clearance rate, infected cell life-span, and viral generation time. Science 1996; 271: 1582-1586.

3. Hammer SM. Increasing choices for HIV therapy. N Engl J Med 2002; 346: 2022-2023.

4. Kiguba MN. Discontinuation and modification of highly active antiretroviral therapy in HIV-infected Ugandans: prevalence and associated factors. J Acquir Immune Defic Syndr 2007; 45: 218-223.

5. Antiretroviral Therapy for Human Immunodeficiency Virus Infection. Mandell Douglas and Bennett's Principles and Practice of Infectious Diseases. $7^{\text {th }}$ ed. Churchill Livingstone, 2009.

6. Price RW. The two faces of HIV infection of cerebrospinal fluid. Trends Microbiol 2000; 8: 387-391.

7. Wang L, Mondal D, La Russa VF, et al. Suppression of clonogenic potential of human bone marrow mesenchymal stem cells by HIV type 1 , putative role of HIV-1 tat protein and inflammatory cytokines. AIDS Res Hum Retroviruses 2002; 18: 917-931.

8. Edén A, Fuchs D, Hagberg L, et al. HIV-1 viral escape in cerebrospinal fluids of subjects on suppressive antiretroviral treatment. J Infect Dis 2010; 202: 1819-1825.

9. Clifford DB. Viral escape in cerebrospinal fluid - an Achilles heel of HIV therapy? J Infect Dis 2010; 202: 1768-1769.

10. Brace I. Questionnaire design: How to Plan, Structure and Write Survey Material for Effective Market Research. Kogan Page Publishers, 2008.

11. Biros M. Research without consent: Current status, 2003. Ann Emerg Med 2003; 42: 550-564.

12. Biros M. Research without consent: Exception from and waiver of informed consent in resuscitation research. Sci Eng Ethics 2007; 13: 361-369.

13. Weinger MB, Slagle J, Jain S, et al. Retrospective data collection and analytical techniques for patient safety studies. Journal of Biomedical Informatics 2003; 36: 106-119.

14. Weiss DJ. Nominal analysis of "variable". Behavior Research Methods 2009; 41: 901-908.

15. Johnson VE. Revised standards for statistical evidence, Proc Nat Acad Sci U S A 2013; 110: 19131-19317.

16. Bolstad WM, Curran JM. Introduction to Bayesian statistics. $3^{\text {rd }}$ ed. John Wiley \& Sons, Hoboken 2017.

17. Sarkies MN, Bowles KA, Skinner EH, et al. Data Collection Methods in Health Services Research. Hospital Length of Stay and Discharge Destination. Appl Clin Inform 2015; 6: 96-109.

18. Mobula L, Barnhart M, Malati C, et al. Long-acting, injectable antiretroviral therapy for the management of HIV infection: an update on a potential game-changer. J AIDS Clin Res 2015; 6: 1-5.

19. Scientific Committee on AIDS. Recommended principles of antiretroviral therapy in HIV disease, 2005. Available at: http://www.aids. gov.hk (Accessed: 26.06.2006).

20. Guidelines for the Use of Antiretroviral Agents in HIV-1-Infected Adults and Adolescents. US Department of Health and Human Services, 2015.

21. Margolis DA, Gonzales-Garcia J, Stellbrink HJ. Long-acting intramuscular cabotegravir and rilpivirine in adults with HIV-1 infection (LATTE-2): 96-week results of a randomized, open-label, phase $2 \mathrm{~b}$, non-inferiority trial. Lancet 2017; 390: 1499-1510.

22. Bartlett JA, Shao JF. Successes, challenges, and limitations of current antiretroviral therapy in low-income and middle-income countries. Lancet Infect Dis 2009; 9: 637-649.

23. Woldmedhin B, Wabe NT. The reason for Regimen change among HIV/IDS patients initiated on first line highly active antiretroviral therapy in southern Ethiopia. N Am J Med Sci 2012; 4: 19-23.

24. Clinical Guidelines. Available at: https://aidsinfo.nih.gov/guidelines (Accessed: 25.02.2019).

25. FDA-Approved HIV Medicines. Available at: https://aidsinfo.nih. gov/understanding-hiv-aids/fact-sheets/21/58/fda-approved-hivmedicines (Accessed: 25.02.2019).
26. Prentice RL. A case-cohort design for epidemiologic cohort studies and disease prevention trials. Biometrika 1986; 73: 1-11.

27. Shy CM. Epidemiology 160/600. Introduction to Epidemiology for Public Health course lectures, 1994-2001. Department of Epidemiology, The University of North Carolina at Chapel Hill. 